\title{
Substance Use Patterns and Schizophrenia Spectrum Disorders: A Retrospective Study of Inpatients at a Community Teaching Hospital
}

\author{
Terence Tumenta ${ }^{\mathrm{a}, \mathrm{c}}$, Oluwatoyin Oladeji ${ }^{\mathrm{a}}$, Manpreet Gilla, Basim Ahmed Khan ${ }^{\mathrm{b}}$, \\ Olaniyi Olayinka ${ }^{\mathrm{a}}$, Chiedozie Ojimba ${ }^{\mathrm{a}}$, Tolulope Olupona ${ }^{\mathrm{a}}$
}

\begin{abstract}
Background: Schizophrenia is one of the chronic mental illnesses, characterized by delusions, hallucinations, disorganized speech, grossly disorganized or catatonic behavior, and cognitive decline. It frequently leads to a lifetime of impairment and disability that span the entire lifespan of the patients. Several epidemiologic studies have shown that schizophrenia spectrum disorders (SSDs) contribute significantly to years lived with disability. Additionally, substance use disorders have been reported to co-occur commonly among patients with SSD (a comorbidity also known as dual diagnosis), attracting notable attention over the past few decades. This dual diagnosis often requires treatment modifications to ensure for best patient outcomes.
\end{abstract}

Methods: This study was a retrospective review of the electronic medical charts. The patients included in the study were discharged from the psychiatric unit of our hospital between July 1, 2017 and October 31, 2017. Patients were included in the study using three inclusion criteria: 1) age $\geq 18$ years; 2 ) had a diagnosis of SSD at discharge; and 3) had urine drug screen performed. Sociodemographic and clinical variables were abstracted. Univariate analysis and summary statistics were performed. Bivariate and multivariate analyses were done via logistic regression models to determine the odds ratios (ORs) and corresponding $\mathrm{P}$ values (P).

Results: A total of $365(52.2 \%)$ patients had a diagnosis of SSD at discharge. Of these, 349 met the inclusion criteria. The age ranged from 19 to 79 years, with a mean age of 42.2 years, and $76.8 \%$ of the patients used substances. Out of the 269 patients who used substances, $199(74 \%)$ used two or more substances. Tobacco use was most prevalent $(62.3 \%)$, followed by cannabis use $(41.5 \%)$, alcohol use $(40.2 \%)$, and cocaine use $(27.4 \%)$. Patients who reported using tobacco, were

Manuscript submitted October 23, 2020, accepted December 8, 2020

Published online December 18, 2020

${ }^{a}$ Department of Psychiatry and Behavioral Sciences, Interfaith Medical Center, Brooklyn, NY, USA

bShifa College of Medicine, Islamabad, Pakistan

${ }^{c}$ Corresponding Author: Terence Tumenta, Department of Psychiatry and Behavioral Sciences, Interfaith Medical Center, Brooklyn, NY, USA.

Email: ttumenta@interfaithmedical.com

doi: https://doi.org/10.14740/jocmr4380 more likely to have comorbid alcohol use $(\mathrm{OR}=7.24 ; \mathrm{P}=0.000)$, cannabis use $(\mathrm{OR}=2.80 ; \mathrm{P}=0.000)$, cocaine use $(\mathrm{OR}=5.00 ; \mathrm{P}=0.000)$, and synthetic cannabis $(\mathrm{K} 2)$ use $(\mathrm{OR}=4.62 ; \mathrm{P}=0.048)$. Results of the multivariate analyses supported the other findings.

Conclusions: Our study found a high association between schizophrenia spectrum disorders and substance use, with three out of four patients with SSD using a substance. This prevalence is higher than previously reported by other studies. Among those who use substances, about three in four use multiple substances. These point to some interaction between the substances and appear to be heavily influenced by significant social determinants of mental health that continue to plague the community. It is important to establish if a patient with schizophrenia has a comorbid substance use disorder, because addressing both generally leads to better patient outcomes.

Keywords: Substance use; Schizophrenia; Schizophrenia spectrum disorders; Mental health; Public health

\section{Introduction}

Schizophrenia is one of the chronic mental illnesses. Patients commonly present with delusions, hallucinations, disorganized speech, grossly disorganized or catatonic behavior, and negative symptoms. It frequently leads to a lifetime of impairment, cognitive decline, and disability that span the entire lifespan of the patients. Several epidemiologic studies have shown that schizophrenia spectrum disorders (SSDs) contribute significantly to years lived with disability [1]. Additionally, substance use disorders (SUDs) have been reported to co-occur commonly among patients with SSD (a comorbidity also known as dual diagnosis), attracting notable attention over the past few decades [2-5]. For example, in one epidemiological study, $47 \%$ of patients with schizophrenia were reported to have a SUD [2]. Patients diagnosed with schizophrenia and other psychotic disorders usually have comorbid SUDs. This dual diagnosis often requires treatment modifications to ensure for best outcomes. This estimate is in-keeping with other studies reporting approximately half of schizophrenia patients have a comorbid SUD $[4,5]$.

Most patients with comorbid SSD and substance use are 
young males and have been shown to have a higher risk for medication noncompliance, multiple hospitalizations, unstable housing and homelessness, human immunodeficiency virus (HIV) infection, suicide, and violent behavior [3]. Regarding specific substances, those who use alcohol are reported to have higher hospital admissions, higher severity of positive symptoms, higher rates of extrapyramidal side effects including tardive dyskinesia, and "relative neuroleptic refractoriness" [2]. There have been studies about the exacerbation of psychotic symptoms, increased hospital admissions, and increased tardive dyskinesia with cannabis use $[2,5]$; while cocaine use reportedly is associated with a higher risk of depression, milder negative symptoms, and a high rate of readmission [2]. Additionally, patients with dual diagnosis have been shown to have a link between current suicide risk, positive symptoms, insight, and first-degree relatives with SUD [6].

Although several studies show an association between SSD and substance use, with the attendant negative health effects, the direction of influence remains unclear. Furthermore, it is uncertain if it is more likely that patients with SSD would use one or a combination of substances (e.g., use of tobacco alone versus tobacco and cannabis). Hence, investigating the pattern of substance use among patients with SSD in different clinical/geographic settings is a crucial step. In this research study, we choose to evaluate the prevalence of substance use and the common combination of substances used by patients diagnosed with SSDs at a community teaching hospital.

\section{Materials and Methods}

\section{Study setting and population}

The study was conducted at Interfaith Medical Center, a community teaching hospital in Central Brooklyn, NY. The hospital serves patients, representing every racial, ethnic, and national origin group in Central Brooklyn, with the majority being Caribbean-Americans and African Americans.

\section{Study design and sample}

This study was a retrospective review of the electronic medical charts. The patients included in the study were discharged from the psychiatric unit of our hospital between July 1, 2017 and October 31, 2017. Patients were included in the study using three inclusion criteria: 1) age $\geq 18$ years old; 2) had a diagnosis of SSD at discharge; and 3) had urine drug screen performed.

\section{Data collection and management}

Data on discharges from the inpatient psychiatric unit were obtained from the director of quality assurance. Data on study variables were manually abstracted from the electronic medical record system and populated into an excel spreadsheet. The data were recoded and exported to STATA software for analy- sis. To maintain HIPAA compliance, no personal identifiable information was recorded.

\section{Choice of variables and categorization}

The variables chosen were hypothesized to influence substance use directly or indirectly in patients with SSD. Sociodemographic characteristics included age, gender, race, employment status, and living arrangement of the patients. The clinical variables included length of stay in the hospital, readmission to the hospital within 30 days, urine drug screen result, and self-reported tobacco use.

Some quantitative variables were recoded into categorical variables. As such, age was recoded into two categories $(0$ $=$ patients aged 18 to 42 years; 1 = patients aged 43 years or older); readmission to the hospital within 30 days was recoded as " 0 " for no readmission within 30 days following discharge and " 1 " for patients that were readmitted within 30 days of discharge; given that the median length of stay was 12 days, length of hospital stay (LOS) was recoded as " 0 " for patients with a hospital stay of 12 days or less, and " 1 " for those with a hospital stay greater than 12 days.

\section{Statistical methods}

Statistical analysis was done using STATA software version 16. The Chi-square test and $t$-test for independent samples were used to compare groups at baseline. A total of 10 variables were included in the baseline analysis and data description. Frequencies were reported for the substances used by the study population.

In patients who reported using tobacco $(62.3 \%)$, bivariate logistic regression analyses were run to determine the odds ratios (ORs) and corresponding P values of co-occurring substance use. We defined statistical significance using a twotailed $\mathrm{P}$ value of less than or equal to 0.05 , for all analyses. A multivariate logistic regression was then performed including all covariates in the unadjusted bivariate models, regardless of statistical significance.

We used the Hosmer-Lemeshow test to verify the goodness-of-fit of our model. The goodness-of-fit model shows that the model cannot be dismissed and therefore leads to the inference that the model fits well.

\section{Ethical issues}

For this study, institutional review board (IRB) approval was obtained. This research was carried out in accordance with the ethical principles of the organization responsible for human subjects, as well as with the Declaration of Helsinki.

\section{Results}

We extracted 365 (52.2\%) of records with a discharge diagno- 
Table 1. Characteristics of the Study Population

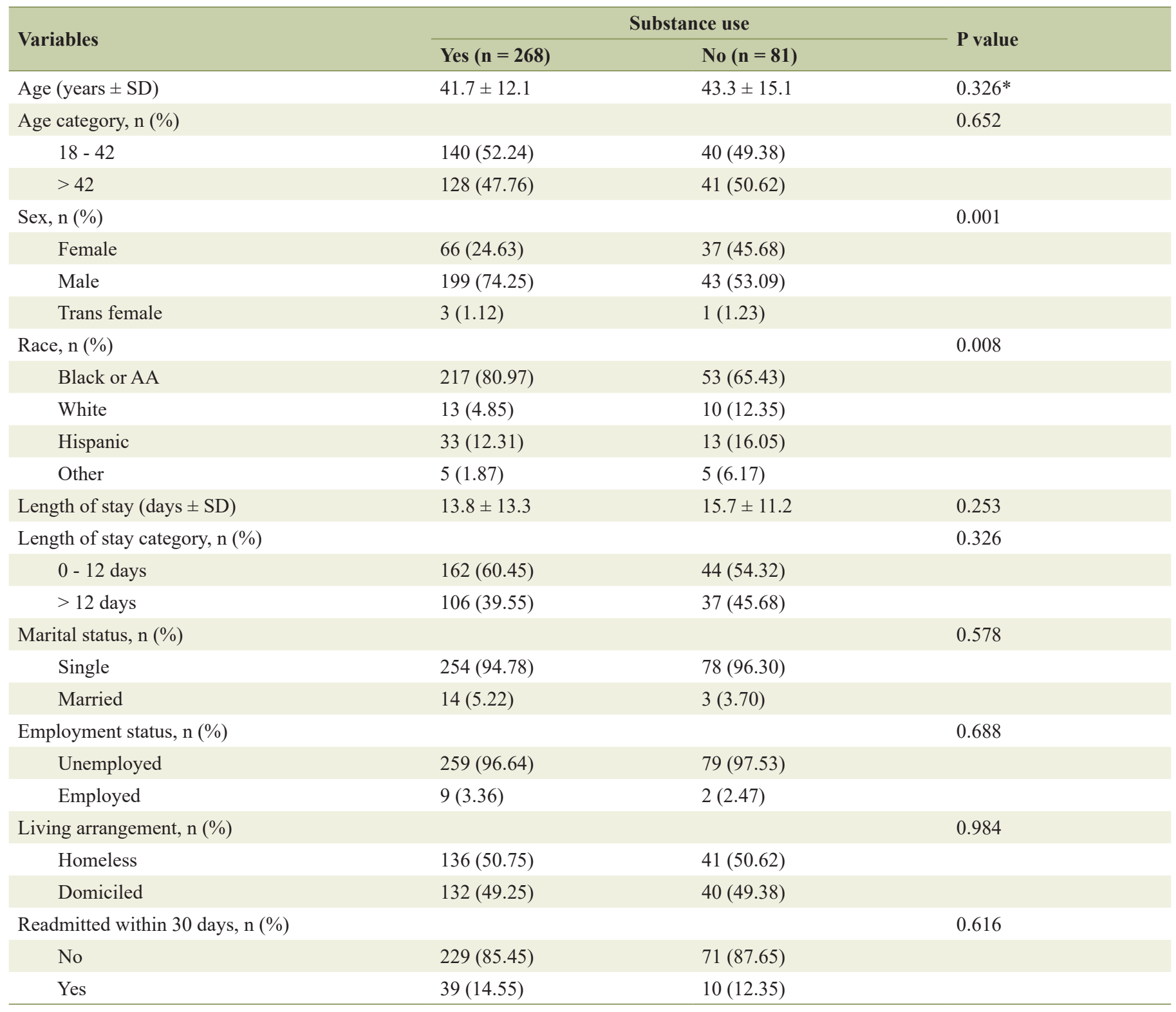

*Based on two sample $t$-test for independent samples. All other analyses in Table 1 are based on $\mathrm{X}^{2}$ test. Note that all patients included in this study were diagnosed with schizophrenia spectrum disorders (SSDs). AA: African American.

sis of SSD out of a total of 698 discharges. Of these, 16 patients were excluded as they did not have a urine drug screen and did not report on their tobacco use. There were 349 patients that were included in the study (Table 1), of which $77.4 \%$ were African American, 6.6\% White, 13.2\% Hispanic, and 2.8\% identified as other race. Of the 349 patients, $76.8 \%$ used substances as evidenced by urine drug screen results and/or selfreported tobacco use. The mean age was 42.2 years, with the youngest being 19 years and the oldest being 79 years. The mean age was 41.7 years for those using substances as compared to 43.3 years in those who did not report any substance use. There was no statistical difference between the young (18 - 42 years) versus the older age group ( $>42$ years), irrespec- tive of whether they used substances or not. The male gender was the most represented, with $69.3 \%$ of the study population, and it was also significantly associated substance use ( $\mathrm{P}$ value $=0.001)$. African Americans used substances more than any other race, though the association was not statistically significant $(\mathrm{P}$ value $=0.008)$. Other factors such as being single, being unemployed, and being homeless, were also found to be nonsignificant in our study populations, with $\mathrm{P}$ values of 0.578 , 0.688 , and 0.984 , respectively.

Among those with substance use, 74\% (199/269) used two or more substances. In the frequency analyses (Table 2), tobacco use was most prevalent $(62.3 \%)$, followed by cannabis use (41.5\%), alcohol use (40.2\%), and cocaine use (27.4\%). In the 
Table 2. Frequency Distribution of the Substances Used in the Study Population

\begin{tabular}{|c|c|c|}
\hline Variable & $\mathbf{N}$ & $\%$ \\
\hline \multicolumn{3}{|l|}{ Alcohol } \\
\hline No & 205 & 59.8 \\
\hline Yes & 138 & 40.2 \\
\hline \multicolumn{3}{|c|}{ Amphetamine } \\
\hline No & 316 & 98.1 \\
\hline Yes & 6 & 1.9 \\
\hline \multicolumn{3}{|c|}{ Benzodiazepine } \\
\hline No & 314 & 96.6 \\
\hline Yes & 6 & 3.4 \\
\hline \multicolumn{3}{|l|}{ Cannabis } \\
\hline No & 189 & 58.5 \\
\hline Yes & 134 & 41.5 \\
\hline \multicolumn{3}{|l|}{ Cocaine } \\
\hline No & 236 & 72.6 \\
\hline Yes & 89 & 27.4 \\
\hline \multicolumn{3}{|c|}{ K2 (synthetic cannabinoids) } \\
\hline No & 217 & 93.1 \\
\hline Yes & 16 & 6.9 \\
\hline \multicolumn{3}{|l|}{ Opioids } \\
\hline No & 312 & 95.7 \\
\hline Yes & 14 & 4.3 \\
\hline \multicolumn{3}{|l|}{ Tobacco } \\
\hline No & 129 & 37.7 \\
\hline Yes & 213 & 62.3 \\
\hline
\end{tabular}

bivariate analyses (Table 3 ), among patients who reported using tobacco, alcohol use $(\mathrm{OR}=7.24$; $\mathrm{P}$ value $=0.000)$, cannabis use $(O R=2.80 ; \mathrm{P}$ value $=0.000)$, cocaine use $(\mathrm{OR}=5.00$; $\mathrm{P}$ value $=0.000)$, and synthetic cannabis $(\mathrm{K} 2)$ use $(\mathrm{OR}=4.62$; $\mathrm{P}$ value $=0.048)$, were all found to be statistically significant. However, after adjusting for all covariates in the multivariate analysis, the only variables significantly associated with tobacco use in our study population were alcohol use, cannabis use, and cocaine use (Table 4).

The Hosmer-Lemeshow test was used to verify the goodness-of-fit of our model (number of groups $=7 ; \chi^{2}=6.64 ; \mathrm{P}$ value $=0.248$ ). The result shows that the model cannot be dismissed and therefore leads to the inference that the model fits well.

\section{Discussion}

This study found that more than three quarters of the patients reported substance use. Of these, more than six out of 10 reported smoking tobacco. Patients with schizophrenia are three times more likely than the general population to start smok-
Table 3. Unadjusted Odds Ratios (ORs), 95\% Confidence Intervals (Cls) and $\mathrm{P}$ Values From Bivariate Logistic Regression Models in Patients Using Tobacco

\begin{tabular}{llll}
\hline Variables & Unadjusted OR & $\mathbf{9 5 \%}$ CI & P value \\
\hline $\begin{array}{l}\text { Alcohol use } \\
\quad \text { Yes vs. No }\end{array}$ & 7.24 & $4.14-12.67$ & 0.000 \\
$\begin{array}{l}\text { Amphetamine use } \\
\quad \text { Yes vs. No }\end{array}$ & 3.09 & $0.36-26.74$ & 0.307 \\
$\begin{array}{l}\text { Benzodiazepine use } \\
\quad \text { Yes vs. No }\end{array}$ & 2.78 & $0.59-13.10$ & 0.196 \\
$\begin{array}{l}\text { Cannabis use } \\
\quad \text { Yes vs. No }\end{array}$ & 2.8 & $1.70-4.59$ & 0.000 \\
$\begin{array}{c}\text { Cocaine } \\
\quad \text { Yes vs. No }\end{array}$ & 5 & $2.60-9.52$ & 0.000 \\
$\begin{array}{l}\text { K2 use } \\
\quad \text { Yes vs. No }\end{array}$ & 4.62 & $1.02-20.99$ & 0.048 \\
\hline $\begin{array}{c}\text { Opioids } \\
\text { Yes vs. No }\end{array}$ & 2.26 & $0.62-8.27$ & 0.218 \\
\hline
\end{tabular}

ing and are five times less likely to stop smoking, with current prevalence rates of up to $80 \%$ [7].

In our study, we found that most patients used multiple substances as well as a significant link between tobacco use and other substances such as alcohol, cannabis, and cocaine. According to Canadian Schizophrenia Guidelines, in people who have schizophrenia and other psychotic disorders, substance use is common [8]. A possible explanation is that the

Table 4. Adjusted Odds Ratios (ORs), 95\% Confidence Intervals (Cls) and $\mathrm{P}$ values From Multivariate Logistic Regression Model in Patients Using Tobacco ${ }^{a}$

\begin{tabular}{llll}
\hline Variables & Adjusted OR & $\mathbf{9 5 \%}$ CI & P value \\
\hline $\begin{array}{l}\text { Alcohol use } \\
\quad \text { Yes vs. No }\end{array}$ & 2.3 & $1.12-4.72$ & 0.023 \\
$\begin{array}{l}\text { Amphetamine use } \\
\quad \text { Yes vs. No }\end{array}$ & 2.35 & $0.20-27.03$ & 0.493 \\
$\begin{array}{l}\text { Benzodiazepine use } \\
\quad \text { Yes vs. No }\end{array}$ & 2.62 & $0.23-29.65$ & 0.436 \\
$\begin{array}{l}\text { Cannabis use } \\
\quad \text { Yes vs. No }\end{array}$ & 2.94 & $1.58-5.48$ & 0.001 \\
$\begin{array}{l}\text { Cocaine use } \\
\quad \text { Yes vs. No }\end{array}$ & 3.4 & $1.45-7.97$ & 0.005 \\
$\begin{array}{l}\text { K2 use } \\
\quad \text { Yes vs. No }\end{array}$ & 2.22 & $0.40-12.37$ & 0.362 \\
$\begin{array}{c}\text { Opioids } \\
\text { Yes vs. No }\end{array}$ & 1.82 & $0.40-8.32$ & 0.442 \\
\hline
\end{tabular}

aAll the covariates in the bivariate analyses in Table 3, were included in the multivariate model regardless of statistical significance. 
ultimate shared influence of substances on the brain reward circuitry affects individuals who use several [9]. The findings in this research like those of other studies, indicate that persons with SSD use substances at a higher rate. In a prevalence study of patients with a psychiatric disorder conducted in Denmark in 2016, of the 53,035 patients with schizophrenia, $37 \%$ $(19,623)$ reported using a substance [10]. Mueser et al reported in their study that alcohol was mostly likely to be abused by patients $(47 \%)$, followed by cannabis $(42 \%)$, stimulants $(25 \%)$, and hallucinogens $(18 \%)$. Sedatives $(7 \%)$ or narcotics $(4 \%)$ were abused by very few patients [11]. In severely mentally ill psychiatric patients, the most common type of SUD, followed by cannabis and cocaine use, was alcohol [12]. The misuse of both alcohol and cannabis has a negative effect on the clinical outcomes of schizophrenia. In the case of cannabis, it was also debated whether schizophrenia would actually be induced, on the basis of a three-to-six-fold dose-dependent rise in the risk of psychosis among cannabis users [7].

Large epidemiologic studies indicate that among people with schizophrenia, the rate of SUDs (excluding nicotine and caffeine use disorders) is $47 \%$ and $44.8 \%$, respectively; with alcohol and cannabis being the most used substances. Moreover, in $60-90 \%$ of people with schizophrenia and other psychotic disorders, cigarette smoking has been documented [8]. Hunt et al conducted a meta-analysis and found that SUDs are extremely prevalent in schizophrenia, and rates over time have remained unchanged. They reported that the prevalence of any SUD was $41.7 \%$. This was followed by illicit drugs with $27.5 \%$, cannabis with $26.2 \%$, alcohol with $24.3 \%$, and stimulant use with $7.3 \%$ of the study population [4]. However, Margolese et al found that patients with ongoing depressive disorder compared to those with SSD (49.6\%) were more likely to smoke cigarettes $(88.9 \%)$ and they had significantly longer cigarette smoking histories (19.1 years for depressive disorders vs. 11.5 years for SSD) $[13,14]$. Nesvag et al looked at the prevalence of SUD in patients diagnosed with schizophrenia, bipolar disorder, and depression. They reported that SUD was prevalent among $25.1 \%$ patients with schizophrenia, $20.1 \%$ patients with bipolar disorder, and $10.9 \%$ patients with depressive illness [10]. Looking at the number of patients using cocaine in our study, the results are in line with other studies which reported that beginning in 1988 and continuing to the present, cocaine use has risen significantly among urban schizophrenic patients. In addition, since the crack epidemic, cocaine appears to be the preferred substance of abuse for schizophrenic patients, surpassing amphetamines [9].

With respect to gender, our study found that males used more substances than females. This finding is similar to that of DeQuardo and colleagues who reported that the rate of substance abuse was much lower in female (20\%) compared to male subjects (48\%). The most widely abused drug, however, was cannabis $(28 \%)$, followed closely by alcohol $(21 \%)$, with slightly less patients abusing cocaine, hallucinogens, and stimulants [15]. For both substance abuse and schizophrenia, gender is an especially significant factor. For alcohol and cannabis in particular, males abused each class of drugs more than females [12]. Verma et al reported in their study of the Asian population looking at patients with a first episode of SSD, that alcohol was the substance most abused. They also stated that substance users were more likely to be males and were also more likely to have a criminal record than those who did not use substances [16].

\section{Limitations}

There are some drawbacks to this study, including those relating to the retrospective study design of medical charts. We were constrained, for instance, by the quality of information given in the charts. To assess whether the patients met criteria for SUD, we did not characterize the substance based on severity, such as mild, moderate, and severe. This was further compounded by using self-reports to determine tobacco use disorder, which is inherently subject to recall bias and underreporting. Another limitation of the study is that we did not look at medical comorbidities in these patients, factors that can also predispose to substance use.

The relatively large sample size is a strength of this analysis, which makes it possible that minor variations were observed between the study variables.

\section{Conclusions}

Patients diagnosed with schizophrenia and other psychotic disorders usually have comorbid SUDs. This dual diagnosis often requires treatment modifications to ensure for best outcomes. Our study found a high association between SSDs and substance use, with three out of four patients with SSD using a substance. This prevalence is higher than previously reported by other studies. Among those who use substances, about three in four use multiple substances. These points to some interaction between the substances and appears to be heavily influenced by significant social determinants of mental health that continue to plague the community. As previously reported by almost all studies if not all, there is a need to modify treatment to accommodate or address substance use comorbidity to achieve a favorable outcome in patients with SSD. This makes it important to establish if a patient with schizophrenia has a comorbid SUD. Psychiatric patients with comorbid SUD are frequently diagnosed later than those with no substance use, therefore delaying effective treatment. Identifying those at high risk so that care is initiated sooner would be of great interest.

\section{Acknowledgments}

For her guidance and insightful suggestions, the authors wish to thank Dr. Noela Co, Director of Quality Assurance, Interfaith Medical Center, Department of Psychiatry and Behavioral Sciences, Brooklyn, NY, USA.

\section{Financial Disclosure}

The authors did not receive any funding and have no financial disclosures. 


\section{Conflict of Interest}

The authors have no conflict of interest.

\section{Informed Consent}

This was a retrospective review of patients' charts. The patients were discharged from Interfaith Medical Center's inpatient psychiatric unit. No personal identifiable information was obtained from study participants.

\section{Author Contributions}

As the primary author of the manuscript, TT has contributed to the study design, data collection, data analysis, helped to write the final draft of the manuscript, and has complete access to all the data in the study. O. Oladeji assisted with the gathering of data and the preparation of the final draft of the manuscript. MG assisted with the gathering of data and the writing of the final draft of the manuscript. BK assisted with gathering of data and writing of the final draft of the manuscript. O. Olayinka contributed to the study's design, assisted with collection of data, and the writing of the final manuscript. $\mathrm{CO}$ helped with data collection and the final manuscript writing. TO contributed to the design of the report and supervised the writing of the manuscript's final draft.

\section{Data Availability}

Any questions should be addressed to the corresponding author about the supporting data availability.

\section{References}

1. Volk DW, Lewis DA. Chapter 105 - Schizophrenia. In: Rosenberg RN, Pascual JM, editor(s). Pascual. Rosenberg's Molecular and Genetic Basis of Neurological and Psychiatric Disease (Fifth Edition), Academic Press. 2015. p. 1293-1299. ISBN 9780124105294.

2. Fowler IL, Carr VJ, Carter NT, Lewin TJ. Patterns of current and lifetime substance use in schizophrenia. Schizophr Bull. 1998;24(3):443-455.

3. Kivimies K, Repo-Tiihonen E, Kautiainen H, Tiihonen J. Comorbid opioid use is undertreated among forensic patients with schizophrenia. Subst Abuse Treat Prev Policy. 2018;13(1):39.

4. Hunt GE, Large MM, Cleary M, Lai HMX, Saunders JB.
Prevalence of comorbid substance use in schizophrenia spectrum disorders in community and clinical settings, 1990-2017: Systematic review and meta-analysis. Drug Alcohol Depend. 2018;191:234-258.

5. Olayinka O, Ojimba C, Alemu B, Olaolu O, Edomias D, Popoola O, Kallikkadan J, et al. Cannabis use in inpatients with schizophrenia spectrum disorders at a community hospital. J Clin Med Res. 2020;12(4):243-250.

6. Adan A, Capella MD, Prat G, Forero DA, Lopez-Vera S, Navarro JF. Executive functioning in men with schizophrenia and substance use disorders. Influence of Lifetime Suicide Attempts. PLoS One. 2017;12(1):e0169943.

7. Thoma P, Daum I. Comorbid substance use disorder in schizophrenia: a selective overview of neurobiological and cognitive underpinnings. Psychiatry Clin Neurosci. 2013;67(6):367-383.

8. Crockford D, Addington D. Canadian Schizophrenia Guidelines: Schizophrenia and Other Psychotic Disorders with Coexisting Substance Use Disorders. Can J Psychiatry. 2017;62(9):624-634.

9. Patkar AA, Alexander RC, Lundy A, Certa KM. Changing patterns of illicit substance use among schizophrenic patients: 1984-1996. Am J Addict. 1999;8(1):65-71.

10. Nesvag R, Knudsen GP, Bakken IJ, Hoye A, Ystrom E, Suren P, Reneflot A, et al. Substance use disorders in schizophrenia, bipolar disorder, and depressive illness: a registry-based study. Soc Psychiatry Psychiatr Epidemiol. 2015;50(8):1267-1276.

11. Mueser KT, Yarnold PR, Rosenberg SD, Swett C, Jr., Miles KM, Hill D. Substance use disorder in hospitalized severely mentally ill psychiatric patients: prevalence, correlates, and subgroups. Schizophr Bull. 2000;26(1):179192.

12. Mueser KT, Yarnold PR, Levinson DF, Singh H, Bellack AS, Kee K, Morrison RL, et al. Prevalence of substance abuse in schizophrenia: demographic and clinical correlates. Schizophr Bull. 1990;16(1):31-56.

13. Margolese HC, Malchy L, Negrete JC, Tempier R, Gill K. Drug and alcohol use among patients with schizophrenia and related psychoses: levels and consequences. Schizophr Res. 2004;67(2-3):157-166.

14. Margolese HC, Carlos Negrete J, Tempier R, Gill K. A 12-month prospective follow-up study of patients with schizophrenia-spectrum disorders and substance abuse: changes in psychiatric symptoms and substance use. Schizophr Res. 2006;83(1):65-75.

15. DeQuardo JR, Carpenter CF, Tandon R. Patterns of substance abuse in schizophrenia: nature and significance. J Psychiatr Res. 1994;28(3):267-275.

16. Verma SK, Subramaniam M, Chong SA, Kua EH. Substance abuse in schizophrenia. A Singapore perspective. Soc Psychiatry Psychiatr Epidemiol. 2002;37(7):326-328. 